\title{
Effect of Managerial Innovation Educational Program for Head Nurses on their Professional Competency
}

\section{Fatma Fouad Elsayed ${ }^{1}$, Nermin Mohamed Huessin ${ }^{2}$ and Fawzia Farouk Elsayed ${ }^{3}$}

(1) Assistant lecturer of Nursing Administration, Faculty of Nursing - Beni seuf University

(2)Professor of Nursing Administration, Faculty of Nursing - Menofia University, (3) Assisstant

Professor of Nursing Administration, Faculty of Nursing - Benha University

\section{Abstract}

Background: Managerial innovation is necessary at the workplace to enhance head nurse's professional competency so head nurses need to adopt with clear and attainable system for managerial innovation in health care sector. The study aimed to assess the effect of managerial innovation educational program for head nurses on their professional competency. Design: A quasi- experimental design was utilized. Setting: The study was conducted at Benha University Hospitals in all medical and surgical buildings. Subjects: Consisted of 55 head nurses. Tools of data collection: Three tools were used in the present study for data collection, innovation knowledge questionnaire, managerial innovation skills questionnaire and professional competency questionnaire, Results: Minority of head nurses (12.7\%) had good level of knowledge at preprogram phase while increased to more than two thirds $(81.8 \% \& 70.9 \%)$ at post program and follow up study phases. The most $(85.5 \% \& 80 \%)$ of head nurses had high level on managerial innovation skills at post program and follow up phases respectively as compared to preprogram phase (14.5\%). Most (81.8\%\& $70.9 \%$ ) of head nurses had good professional competency at post program and follow up, respectively. Conclusion: There was a highly positive statistically significant correlation between head nurses' managerial innovation knowledge, skills, and level of professional competency at post and follow up program phases. Recommendations: Hospital management can organize educational programs for refreshing and increasing knowledge and skills about nurses' innovation as well as emphasizing managerial innovation skills of head nurses.

\section{Keywords: Educational program, Head nurses, Managerial innovation, Professional competency}

\section{Introduction}

The world is changing with really remarkable speed. On the other hand, there is a need to improve efficiency and quality of work, service delivery by introducing innovation in the hospital. Organizations have several objectives including competitiveness, high profit and long-term survival, today, with the complexity of competition; innovation is considered one of the main advantages of organizations. The emergence of innovation not only enables organizations to gain a competitive advantage over competitors but also provides them with a useful tool to improve organizational performance (Kelly, 2017 ; Knoff, 2019).

Innovation is the adoption or generation of new ideas, objects or practices. It is a means for an organization to change, either as pre-emptive actions to affect the environment or in response to changes in the external environment. Therefore, the innovation here is generally defined as covering various components, including new organizational structures, new process technologies, or services or new programs or plans for an organization's members (Saleh\&Al-Dhaafri, 2019).

Managerial innovation is the generation and implementation of a management practice, process, structure, or technique that is new to the state of the art and is intended to further organizational goals. Additionally managerial innovation considered as marked departure from traditional management principles, processes, and practices or a departure from customary organizational forms that significantly alters the 
way the work of management is performed (Alzuod, 2019).

Competency is the application and demonstration of appropriate knowledge, skills, and behaviors in a clinical setting. Head nurse competency is not only the performance or behaviors itself. But the repertoire of abilities, actions, procedure, and responses accessible that allows arranging of work requirements to be done successfully. This competency is not the same thing as performances, but it's what empowers the performances to happen and competency then is a concept that represents a constellation of the characteristics and abilities of the manger that leads to effective performance (Fukada, 2018 ; Markazi, et al, 2019).

The head nurse role is currently seen as one of the hardest, most complex roles in healthcare. Also head nurse is responsible for translating strategic goals and objectives formulated at the operational level into practice; thus, the position of head nurse requires an ability to interpret general concepts and integrate them into specific clinical and management performance, while simultaneously determining and monitoring outcomes (Chase, 2020).

In addition, the head nurse role provides not only administrative and clinical leadership, but also has 24-hour accountability for all patient care activities on the unit. The role of the head nurse in the acute care nursing area is pivotal in the development and retention of staff, as well as overall unit productivity. In order to do this role effectively and efficiently requires certain managerial innovation skills to improve their competencies (Gunawan \& Aungsuroch, 2017).

Head nurses are looking for new rules of the road to give them a competitive edge and fuel new problem solving skills. To make effective sense of unfamiliar situations and complex challenges, they must have a grasp of the whole of the situation, including its variables, unknowns, and mysterious forces. This requires skills beyond everyday analysis, it requires managerial innovation. Head nurses should enjoy appropriate skill level and knowledge related to managerial innovation that enhance their professional competency (Rabea, 2017).

\section{Significance of the study}

The healthcare environment and nursing services are undergoing a rapid change. So head nurse should play a key role in providing high quality and safe services to patients in this continuously changing environment. For nurse managers to successfully fulfill these vital roles, their innovation skills need to be strengthened. Innovation is critical to professional competency of nurse managers, and organization need development programs to improve creative problem solving, communication, motivation of others and creativity of nurse managers.

The competency can be achieved through enhancing managerial innovation and creativity skills for head nurses. By research of nurse managers working at Benha university hospital do routine work without any change or innovation, don't motivate or support staff nurse to think creatively, not receive any educational program about the managerial innovation and its effect on professional competency. So this study will be conduct assess the effect of managerial innovation educational program for head nurses on their professional competency.

\section{Aim of the study:}

This study aimed to assess the effect of managerial innovation educational program for head nurses on their professional competency

\section{Subjects and methods:}

The methodology of the current study was portrayed according to four following designs: Technical design, operational design, administrative design, and statistical design.

\section{Research design:}

A quasi-experimental design was utilized to conduct this study.

\section{Setting:}


The study was conducted at Benha University Hospital in all medical and surgical units, the total number of beds at this hospital is (880) beds. The hospital composed of three separated buildings; medical building with capacity (478 beds), surgical building with capacity (384 beds) and ophthalmology building with capacity (18 beds). This hospital contains 34 medical units and 21 surgical units

\section{Subjects:}

The subject consisted of all head nurses (55); Medical Units (34) and Surgical Units (21) who are working at previous mentioned setting during the time of data collection and accept to participate in the study and distributed as one head nurse in each unit.

\section{Tools of data collection:}

The data for the study was collected by using the following three tools:

\section{(1)Managerial Innovation Knowledge}

\section{Questionnaire:}

It was developed by the researcher through reviewing the related literature (Wately, 2016., Rashed, 2017., Ebrahim, 2018) to assess head nurses' knowledge regarding managerial innovation. It consisted of two parts:

Part (1): personal characteristics of the study subjects (gender, age, educational qualifications, marital status, department, and years of experiences)

Part (2): It included 46 items to assess head nurses knowledge regarding managerial innovation in the form of multiple choice matching and true or false questions about related to (cognitive flexibility, problem solving, risk taking, innovation, motivation, emotional intelligence, learning, keeping goal and direction, and communication).

\section{Scoring system}

The questions were scored as "1" for correct, and "zero" for incorrect so the total scores (46). Total knowledge score was calculated as follow; poor $>60 \%$ that equals ( $\leq 28$ degree), average from $60 \%$ - $<75 \%$ (>28 - <35 degree) score and good $\geq 75 \%$ that equals ( $\geq 35$ degree).
(2)Managerial

Innovation

Skills

\section{Questionnaire:}

It was developed by the researcher through reviewing the related literature (Dacko \& szczepansk, 2014, Maia\& Cristina, 2015, Abdelazeem, 2015, Rashed, 2017 ). It consisted of different items to assess head nurses' managerial innovation skills through the program.

It consisted of (60) items regarding to cognitive flexibility skill (6 items), problem solving skill (7 items), risk taking skill (7 items), innovation skill (11), motivation skill (7 items), emotional intelligence skill (8 items), learning skill (5 items), keeping goal and direction skill(4 items), and communication skill (5 items).

\section{Scoring system}

Head nurses responses were scored based on 3 points of Likert scale as follows: (3) always (2) sometimes (1) never. The total scores (180) are summed up and graded according to the following scores; low level of managerial innovation skills $\leq$ $60 \%$ that equals ( $\leq 108$ degree), moderate level of managerial innovation skill $\geq 60 \%-<75$ that equals ( $\geq 109-\leq 135$ degree) and high level of managerial innovation skill $\geq 75 \%$ that equals( $\geq 135$ degree).

\section{(3) Professional competency questionnaire:}

Structured questionnaire developed by the researcher through reviewing the related literature (American Organization of Nurse Executives, 2015, Rabea,2017, Goktepe, 2018) to assess head nurses professional competency. It consisted of (48) items divided into 7 main dimension; Promote nursing staff retention ( 9 items), Facilitate nursing staff development (7 items), Perform supervisory responsibilities (10 items), Ensure patient safety \&Quality care (5 items), Conduct daily unit operations (5 items), Facilitate interpersonal \&organizational communication (5 items), SelfDevelopment (7 items).

\section{Scoring system}

Head nurses response was scored based on 3 points of likert scale as follows: (3) always (2) sometimes (1) never. The total scores (144) are summed up and graded according to the following 
scores; low level of professional competency

$<60 \%$ that equals $<86$ degree), moderate level of professional competency $\geq 60 \%-<75 \%$ that equals $(\geq 86<108$ degree) and high level of professional competency $\geq 75 \%$ that equals ( $\geq 108$ degree).

\section{Tools' Validity:}

The tools were tested for validity questionnaire (face and content) through distributing the format to a panel of five experts from nursing administration departments at various Faculties of Nursing. (2) Assistant Professor from Cairo University , (2) Assistant Professor from Benha University (1) Assistant Professor from Menoufia University, the necessary modifications were done according to jury comments, the modifications were done in the light of their valuable comments such as modify some words to give the right meaning for the phrases which were not clear.

\section{Pilot study:}

Pilot study was carried out in order to ascertain the clarity and applicability of the study tools. The pilot study was tested on $10 \%$ of sample (5). Head nurses who shared in the pilot study were included in the main study sample. It was also served in estimating the time needed for filling the tools. The time needed for answering knowledge questionnaire sheet "25-30" minutes, observing the actual skill level of head nurses "15-20" and professional competency questionnaire"15-20" Minutes.

\section{Tools' Reliability:}

The reliability of questionnaires sheets was tested for the internal consistency using alpha coefficient as follow; managerial innovation knowledge questionnaire: Cronbach's alpha $=0.94$, managerial innovation skills questionnaire: Cronbach's alpha $=0.91$ and professional competency questionnaire: Cronbach's alpha $=0.92$

\section{Field Work:}

\section{Assessment phase:}

The data was collected to assess head nurses' knowledge, skills regarding managerial innovation and to assess head nurses' professional competency before implementation of the educational program through using of the different tools of data collection in the available hospital classroom and during their work hours. The questionnaire sheets were distributed to head nurses, the investigator was presented all the time during filling the questionnaire sheets for any clarification as needed then the investigator checked each one to ensure its completeness. The investigator was observing head nurses for managerial innovation skills of 3-4 head nurses per day.

\section{Planning phase:}

This phase started from December (2019) to January (2020). An educational program was developed based on determined needs and relevant review of literature. Different instructional strategies, methods of teaching, media and methods of evaluation were selected to suit the learner's needs and achieve the objectives and content of the program. The teaching sessions were achieved by using available resources, relevant content and instructional strategies for each session. Different methods of teaching were used such as lecture, group discussion, brain storming and hand out prepared by the investigator and distributed to all head nurses.

\section{Implementation phase:}

Data of the current study were collected from February 2020 and continued to a period of two months. The educational program was consisted of (14) hours distributed as theoretical session (10) hours and practical session (4) hours. The program took about (7) sessions, the duration of each session was two hours depending on workload and including periods of discussion according to their achievement, progress and feedback. It started at $12.00 \mathrm{pm}$ to $2.00 \mathrm{pm}$, the beginning of first session an orientation to the education program. Feedback was given in the beginning of each session about the previous one 
and at the ending of each session about the current session.

\section{Evaluation phase:}

During this phase the effect of the education program was evaluated. Immediate evaluation included, immediate post program implementation for all subjects using the same tools which were used before the program. Follow up after three months of program implementation, all the study tools were applied to all head nurses to test the follow up gain in the head nurses knowledge, skills regarding to managerial innovation and change in level of head nurses' professional competency.

\section{Ethical Considerations:}

At the interview with head nurses to collect data, they were informed about the purpose and benefits of the study, their participation was voluntary, and they have the right to refuse to participate in the study without giving any reason. In addition, confidentiality and anonymity of the subjects were assured through coding of all data.

\section{Statistical analysis}

Data were verified prior to computerized entry. The Statistical Package for (SPSS version 25.0) was used for that purpose, followed by data analysis and tabulation. For numerical values, the mean and standard deviations were calculated. Descriptive statics were applied ( eg., mean, standard deviation, frequency and percentages, chi-square $\left(\mathrm{X}^{2}\right)$, test of significance (paired (t) test, Pearson correlation coefficients were used investigation of relationships among scores. The P- value is the probability of error that indicate significance of results through observed difference.

\section{Results}

Table (1) shows that more three fifths $(60 \%)$ of studied head nurses their age between 30 $<40$ with mean $(35.83 \pm 6.30)$. As far as their gender, the majorities $(89.1 \%)$ of them were female; about their marital status the majority $(83.6 \%)$ were married. In relation to their educational qualification about two thirds of them $(67.3 \%)$ had a baccalaureate of nursing, and about half of them $(49.1 \%)$ had $10-<20$ years of experience in nursing and all head nurses didn't attend any managerial innovation training courses.

Figure (1) indicating that, the program had a greater effect on improving head nurse's knowledge through post and follow up phases compared with preprogram phase; most of head nurses $(81.8 \%$ \& $70.9 \%$ ) had good level of knowledge during immediate post program and follow up phases respectively as compared with preprogram.

Table (2) summarize that there was a highly statistical difference improvement of head nurses knowledge about managerial innovation between pre and post program phases and between preprogram and follow up phase; that is the educational program has an effect on improving of head nurses knowledge regards to managerial innovation.

Figure (2) revealed that, more than two third $(85.5 \% \& 80 \%)$ of head nurses had high level on managerial innovation skills at post program and follow up phases respectively as compared to preprogram phase $(60 \%)$.

Table(3) shows that there was a highly statistical significant difference improvement of head nurses' related to managerial innovation skills between pre and post program and between preprogram and follow up phase.

Figure (3) demonstrate that, more than two thirds $(81.8 \% \& 70.9 \%)$ of head nurses had good professional competency at post program and follow up phases respectively as compared to preprogram (7.3) phase.

Table (4) shows that there was a highly statistical significant difference improvement of head nurses related to professional competency dimensions between pre and post program and between preprogram and follow up phase.

Table (5) clarifies that a there was highly positive statistical significant correlation between 
head nurses' managerial innovation knowledge and their professional competency in post program phase and there was a positive statistical significant correlation between head nurses' managerial innovation knowledge score and their professional competency in follow up phase.

Table(6) shows that, there was highly positive statistical significant correlation between head nurses' managerial innovation skills and their professional competency in post program phase and there was a positive statistical significant correlation between head nurses' managerial innovation skills and their professional competency in follow up phase. 
Table (1): Frequency distribution of head nurse regarding their personal characteristics $(n=55)$

\begin{tabular}{|c|c|c|}
\hline Personal characteristics & No & $\%$ \\
\hline \multicolumn{3}{|l|}{ Gender } \\
\hline Female & 49 & 89.1 \\
\hline Male & 6 & 10.9 \\
\hline \multicolumn{3}{|l|}{ Age } \\
\hline$<30$ & 8 & 14.5 \\
\hline $30-<40$ & 33 & 60.0 \\
\hline $40+$ & 14 & 25.5 \\
\hline Mean \pm SD & \multicolumn{2}{|c|}{$35.83 \pm 6.30$} \\
\hline \multicolumn{3}{|c|}{ Level of educational qualifications } \\
\hline Diploma in nursing & 8 & 14.5 \\
\hline Associated degree in nursing & 2 & 3.6 \\
\hline BSc in nursing & 37 & 67.3 \\
\hline Master degree in nursing & 8 & 14.5 \\
\hline \multicolumn{3}{|l|}{ Marital status } \\
\hline Married & 46 & 83.6 \\
\hline Not Married & 9 & 16.4 \\
\hline \multicolumn{3}{|l|}{ Years of Experience } \\
\hline$<10$ & 17 & 30.9 \\
\hline $10-<20$ & 27 & 49.1 \\
\hline $20+$ & 11 & 20.0 \\
\hline Mean \pm SD & $13.87 \pm 6.60$ & \\
\hline \multicolumn{3}{|l|}{ Training courses } \\
\hline No & 55 & 100.0 \\
\hline
\end{tabular}

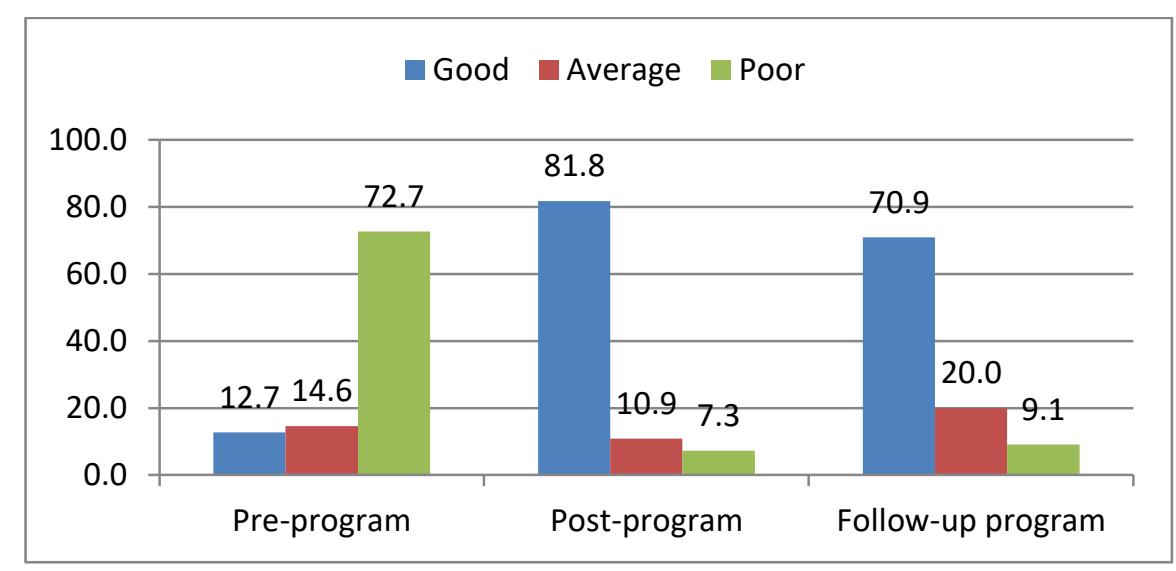

Figure (1): Head nurses' levels regard managerial innovation knowledge through the program phases 
Table (2): Mean and standard deviation of head nurses' total knowledge regarding to managerial innovation through program phases

\begin{tabular}{|c|c|c|c|c|c|c|c|c|c|c|c|c|}
\hline $\begin{array}{l}\text { Managerial } \\
\text { innovation } \\
\text { knowledge }\end{array}$ & $\begin{array}{r}\text { P } \\
\text { pro } \\
\text { Mean }\end{array}$ & $\begin{array}{l}\text { re } \\
\text { ram }\end{array}$ & $\begin{array}{r}\text { P } \\
\text { prog } \\
\text { Mean }\end{array}$ & $\begin{array}{l}\text { st } \\
\text { ram } \\
\text { SD }\end{array}$ & Mean & v-up & t1 & $\begin{array}{l}\text { p- } \\
\text { value }\end{array}$ & t2 & $\begin{array}{l}\text { p- } \\
\text { value }\end{array}$ & t3 & $\begin{array}{l}\text { p- } \\
\text { value }\end{array}$ \\
\hline $\begin{array}{l}\text { Cognitive } \\
\text { Flexibility }\end{array}$ & .90 & $\begin{array}{c} \pm 0.7 \\
2\end{array}$ & 2.65 & $\begin{array}{c} \pm 0.6 \\
7\end{array}$ & 2.67 & \pm 0.72 & 13.66 & $\begin{array}{c}.000 * \\
*\end{array}$ & .163 & .871 & 13.088 & $\begin{array}{c}.000 * \\
*\end{array}$ \\
\hline $\begin{array}{l}\text { Problem } \\
\text { solving }\end{array}$ & 1.67 & $\begin{array}{c} \pm 0.8 \\
8\end{array}$ & 5.40 & $\begin{array}{c} \pm 1.0 \\
2\end{array}$ & 5.21 & \pm 1.11 & 19.2 & $\begin{array}{c}.000 * \\
*\end{array}$ & 1.010 & .317 & 19.968 & $\begin{array}{c}.000 * \\
*\end{array}$ \\
\hline Risk taking & .41 & $\begin{array}{c} \pm 0.4 \\
9\end{array}$ & 1.63 & $\begin{array}{c} \pm 0.5 \\
2\end{array}$ & 1.52 & \pm 0.53 & 13.17 & $\begin{array}{c}.000 * \\
*\end{array}$ & 1.030 & .308 & 11.559 & $\begin{array}{c}.000 * \\
*\end{array}$ \\
\hline Innovation & 4.10 & $\begin{array}{c} \pm 1.8 \\
0\end{array}$ & $\begin{array}{c}12.5 \\
4\end{array}$ & $\begin{array}{c} \pm 2.4 \\
4\end{array}$ & 11.70 & \pm 2.27 & 19.72 & $\begin{array}{c}.000 * \\
*\end{array}$ & 1.702 & .095 & 20.086 & $\begin{array}{c}.000 * \\
*\end{array}$ \\
\hline Motivation & 2.09 & $\begin{array}{c} \pm 1.1 \\
9\end{array}$ & 5.29 & $\begin{array}{c} \pm 1.1 \\
1\end{array}$ & 5.29 & \pm 0.85 & 15.48 & $* * 000$ & .000 & 1.000 & 16.136 & $\begin{array}{c}.000 * \\
*\end{array}$ \\
\hline $\begin{array}{l}\text { Emotional } \\
\text { intelligence }\end{array}$ & 1.72 & $\begin{array}{c} \pm 0.8 \\
2\end{array}$ & 4.03 & $\begin{array}{c} \pm 1.1 \\
5\end{array}$ & 3.72 & \pm 1.25 & 11.69 & $\begin{array}{c}.000 * \\
*\end{array}$ & 1.316 & .194 & 10.208 & $\begin{array}{c}.000 * \\
*\end{array}$ \\
\hline Learning & 1.38 & $\begin{array}{c} \pm 1.0 \\
9\end{array}$ & 3.34 & $\begin{array}{c} \pm 0.9 \\
6\end{array}$ & 3.10 & \pm 0.93 & 9.93 & $\begin{array}{c}.000 * \\
*\end{array}$ & 1.478 & .145 & 9.465 & $\begin{array}{c}.000 * \\
*\end{array}$ \\
\hline $\begin{array}{l}\text { Keeping } \\
\text { goal or } \\
\text { direction }\end{array}$ & 1.03 & $\begin{array}{c} \pm 0.7 \\
9\end{array}$ & 2.72 & $\begin{array}{c} \pm 0.4 \\
8\end{array}$ & 2.63 & \pm 0.61 & 12.34 & $\begin{array}{c}.000 * \\
*\end{array}$ & 0.896 & .374 & 11.146 & $\begin{array}{c}.000 * \\
*\end{array}$ \\
\hline $\begin{array}{l}\text { Communica } \\
\text { tion }\end{array}$ & .65 & $\begin{array}{c} \pm 0.5 \\
8\end{array}$ & 1.80 & $\begin{array}{c} \pm 0.4 \\
4\end{array}$ & 1.76 & \pm 0.42 & 11.62 & $\begin{array}{l}.000 * \\
*\end{array}$ & 0.531 & .598 & 10.467 & $\begin{array}{c}.000 * \\
*\end{array}$ \\
\hline $\begin{array}{l}\text { Total } \\
\text { knowledge }\end{array}$ & & $\begin{array}{r}4.12 \pm \\
2.08\end{array}$ & & $\begin{array}{l}43 \pm \\
5.72\end{array}$ & 37.6 & \pm 5.42 & 29.21 & $\begin{array}{c}0.000 \\
* *\end{array}$ & 1.81 & 0.076 & 31.73 & $\begin{array}{c}0.000 \\
* *\end{array}$ \\
\hline
\end{tabular}

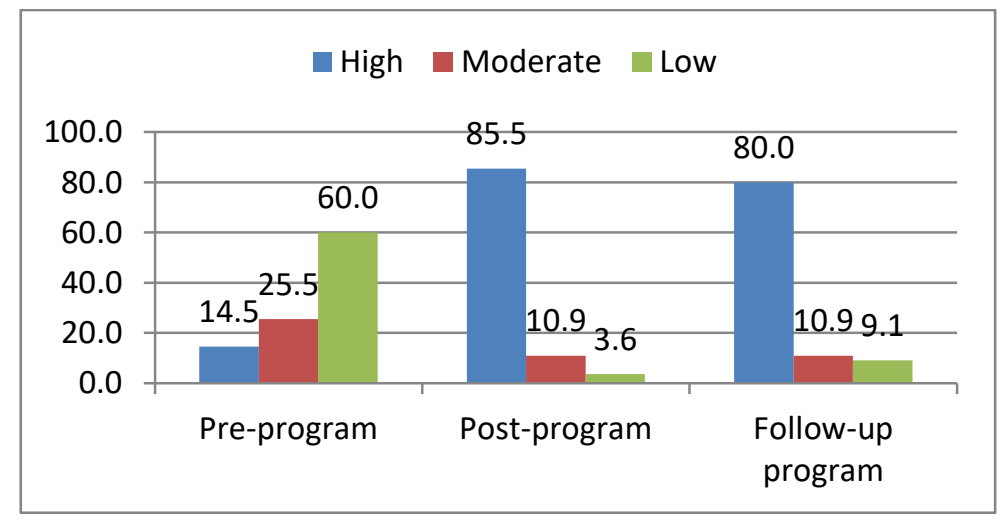

Figure (2): Head nurses' levels regard managerial innovation skills throughout the program phases 
Table (3): Mean and standard deviation of head nurses skills regarding to managerial innovation through program phases

\begin{tabular}{|c|c|c|c|c|c|c|c|c|c|c|c|c|}
\hline \multirow{2}{*}{$\begin{array}{l}\begin{array}{l}\text { Managerial } \\
\text { innovation } \\
\text { skills }\end{array} \\
\end{array}$} & \multicolumn{2}{|c|}{$\begin{array}{c}\text { Pre } \\
\text { program }\end{array}$} & \multicolumn{2}{|c|}{$\begin{array}{c}\text { Post } \\
\text { program }\end{array}$} & \multicolumn{2}{|c|}{ Follow-up } & \multirow[t]{2}{*}{ t1 } & \multirow[t]{2}{*}{$\begin{array}{l}\text { p- } \\
\text { value }\end{array}$} & \multirow[t]{2}{*}{ t2 } & \multirow[t]{2}{*}{$\begin{array}{l}\text { p- } \\
\text { value }\end{array}$} & \multirow[t]{2}{*}{ t3 } & \multirow[t]{2}{*}{$\begin{array}{l}p \text { - } \\
\text { value }\end{array}$} \\
\hline & Mear & \pm SD & Mean & \pm SD & Mean & & & & & & & \\
\hline $\begin{array}{l}\text { Cognitive } \\
\text { Flexibility } \\
\text { Skill }\end{array}$ & $\begin{array}{c}10 . \\
36\end{array}$ & .86 & $\begin{array}{r}20 . \\
12\end{array}$ & $\begin{array}{r}1.6 \\
6\end{array}$ & $\begin{array}{r}19.1 \\
4\end{array}$ & 1.80 & 38.61 & .000 & 2.72 & .009 & $\begin{array}{r}32.31 \\
2\end{array}$ & .000 \\
\hline $\begin{array}{l}\text { Problem } \\
\text { solving } \\
\text { skill }\end{array}$ & $\begin{array}{r}10 . \\
38\end{array}$ & $\begin{array}{r}1.0 \\
2\end{array}$ & $\begin{array}{r}19 . \\
25\end{array}$ & $\begin{array}{r}2.1 \\
3\end{array}$ & $\begin{array}{r}18.6 \\
5\end{array}$ & 2.08 & 27.45 & .000 & 1.47 & .145 & $\begin{array}{r}28.23 \\
8\end{array}$ & .000 \\
\hline $\begin{array}{l}\text { Risk } \\
\text { taking skill }\end{array}$ & $\begin{array}{r}9.3 \\
4\end{array}$ & $\begin{array}{r}1.1 \\
7\end{array}$ & $\begin{array}{r}18 . \\
85\end{array}$ & $\begin{array}{r}2.0 \\
8\end{array}$ & $\begin{array}{r}18.8 \\
5\end{array}$ & 2.59 & 31.79 & .000 & .000 & $\begin{array}{r}1.00 \\
0\end{array}$ & $\begin{array}{r}24.94 \\
0\end{array}$ & .000 \\
\hline $\begin{array}{l}\text { Innovation } \\
\text { skill }\end{array}$ & $\begin{array}{c}14 . \\
56\end{array}$ & $\begin{array}{r}1.4 \\
8\end{array}$ & $\begin{array}{r}29 . \\
90\end{array}$ & $\begin{array}{r}2.8 \\
8\end{array}$ & $\begin{array}{r}29.8 \\
0\end{array}$ & 2.71 & 34.41 & .000 & 0.20 & .840 & $\begin{array}{r}36.34 \\
6\end{array}$ & .000 \\
\hline $\begin{array}{l}\text { Motivation } \\
\text { skill }\end{array}$ & $\begin{array}{c}11 . \\
16\end{array}$ & $\begin{array}{r}1.6 \\
4\end{array}$ & $\begin{array}{r}19 . \\
30\end{array}$ & $\begin{array}{r}1.7 \\
3\end{array}$ & $\begin{array}{r}19.1 \\
2\end{array}$ & 1.99 & 25.85 & .000 & 0.53 & .594 & $\begin{array}{r}21.48 \\
8\end{array}$ & .000 \\
\hline $\begin{array}{l}\text { Emotional } \\
\text { intelligenc } \\
\text { e skill }\end{array}$ & $\begin{array}{r}13 . \\
14\end{array}$ & $\begin{array}{r}1.3 \\
9\end{array}$ & $\begin{array}{r}21 . \\
69\end{array}$ & $\begin{array}{r}2.2 \\
7\end{array}$ & $\begin{array}{r}21.9 \\
2\end{array}$ & 2.00 & 24.97 & .000 & .711 & .480 & $\begin{array}{r}27.94 \\
1\end{array}$ & .000 \\
\hline $\begin{array}{l}\text { Learning } \\
\text { skill }\end{array}$ & $\begin{array}{r}8.0 \\
1\end{array}$ & $\begin{array}{r}2.2 \\
7\end{array}$ & $\begin{array}{r}13 . \\
72\end{array}$ & $\begin{array}{r}1.9 \\
5\end{array}$ & $\begin{array}{r}13.6 \\
3\end{array}$ & 1.55 & 13.97 & .000 & $\begin{array}{r}0.27 \\
5\end{array}$ & .784 & $\begin{array}{r}15.56 \\
4\end{array}$ & .000 \\
\hline $\begin{array}{l}\text { Keeping } \\
\text { goal or } \\
\text { direction } \\
\text { skill }\end{array}$ & $\begin{array}{r}5.6 \\
3\end{array}$ & $\begin{array}{r}1.0 \\
7\end{array}$ & $\begin{array}{r}11 . \\
14\end{array}$ & $\begin{array}{r}1.3 \\
2\end{array}$ & $\begin{array}{r}10.6 \\
0\end{array}$ & 1.63 & 22.52 & .000 & $\begin{array}{r}1.83 \\
8\end{array}$ & .072 & $\begin{array}{r}20.27 \\
9\end{array}$ & .000 \\
\hline $\begin{array}{l}\text { Communic } \\
\text { ation skill }\end{array}$ & $\begin{array}{r}7.5 \\
4\end{array}$ & $\begin{array}{r}1.4 \\
3\end{array}$ & $\begin{array}{r}11 . \\
12\end{array}$ & $\begin{array}{r}1.3 \\
8\end{array}$ & $\begin{array}{r}10.8 \\
3\end{array}$ & 1.42 & 12.41 & .000 & $\begin{array}{r}1.09 \\
1\end{array}$ & .280 & $\begin{array}{r}12.22 \\
2\end{array}$ & .000 \\
\hline Total & $\begin{array}{r}89 . \\
85\end{array}$ & $\begin{array}{r}4.2 \\
6\end{array}$ & $\begin{array}{r}165 \\
.14\end{array}$ & $\begin{array}{r}8.1 \\
4\end{array}$ & $\begin{array}{r}162 . \\
81\end{array}$ & $\begin{array}{r}10.0 \\
1\end{array}$ & 65.86 & .000 & $\begin{array}{r}1.25 \\
7\end{array}$ & .214 & $\begin{array}{r}46.93 \\
4\end{array}$ & .000 \\
\hline
\end{tabular}




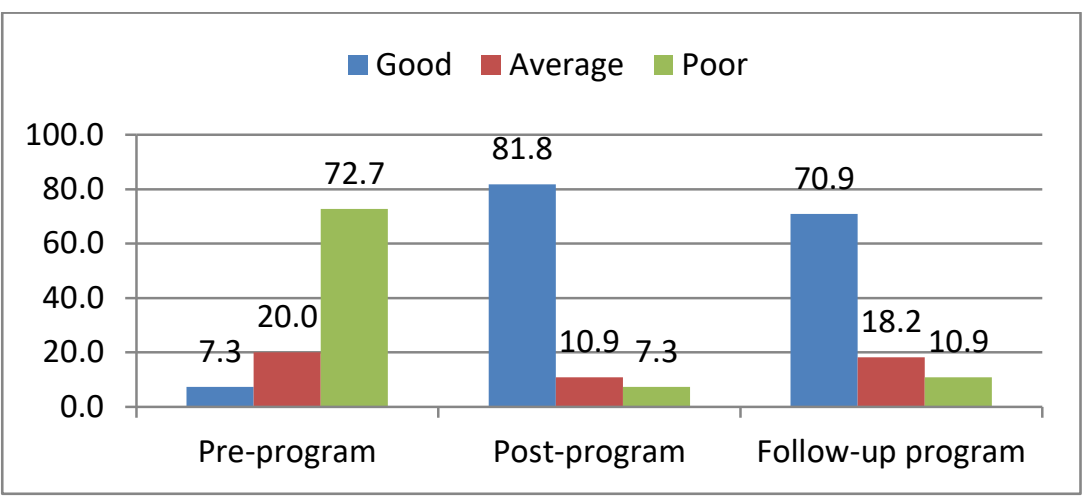

Figure (3): Professional competency level of head nurses through the program phases

Table (4): Mean and standard deviation regarding professional competency through program phases

\begin{tabular}{|c|c|c|c|c|c|c|c|c|c|c|c|c|}
\hline \multirow{2}{*}{$\begin{array}{l}\text { Professional } \\
\text { Competency dimensi }\end{array}$} & \multirow{2}{*}{\multicolumn{2}{|c|}{$\begin{array}{l}\text { Pre Progra } \\
\text { Mean } \pm \text { SD }\end{array}$}} & \multirow{2}{*}{\multicolumn{2}{|c|}{\begin{tabular}{|l|} 
Post Progra \\
Mean $\pm \mathrm{SD}$ \\
\end{tabular}}} & \multirow{2}{*}{\multicolumn{2}{|c|}{$\frac{\text { Follow-up }}{\text { Mean } \pm \text { SD }}$}} & \multirow{3}{*}{$\begin{array}{l}\mathbf{t 1} \\
19.41\end{array}$} & \multirow{3}{*}{$\begin{array}{l}\text { p-value } \\
.000\end{array}$} & \multirow{3}{*}{$\begin{array}{l}\mathbf{t 2} \\
0.16\end{array}$} & \multirow{3}{*}{$\begin{array}{c}\text { p-valu } \\
.872\end{array}$} & \multirow{3}{*}{$\begin{array}{l}\mathbf{t 3} \\
20.23\end{array}$} & \multirow{3}{*}{$\begin{array}{l}\text { p-value } \\
.000\end{array}$} \\
\hline & & & & & & & & & & & & \\
\hline $\begin{array}{l}\text { Promote nursing staff } \\
\text { Retention }\end{array}$ & 13.29 & 3.12 & 24.43 & 2.70 & 24.34 & 2.68 & & & & & & \\
\hline $\begin{array}{l}\text { Facilitate nursing staff } \\
\text { Development }\end{array}$ & 11.36 & 2.61 & 19.90 & 1.35 & 18.72 & 2.39 & 21.86 & .000 & 3.36 & .011 & 17.16 & .000 \\
\hline $\begin{array}{l}\text { Perform } \\
\text { supervisory responsib }\end{array}$ & 17.32 & 2.10 & 28.52 & 2.08 & 28.40 & 2.06 & 32.31 & .000 & 0.33 & .736 & 28.14 & .000 \\
\hline $\begin{array}{l}\text { Ensure patient safety } \\
\text { Quality care }\end{array}$ & 7.29 & 1.49 & 13.38 & 1.97 & 13.76 & 1.47 & 18.09 & .000 & 1.172 & .246 & 21.72 & .000 \\
\hline $\begin{array}{l}\text { Conduct daily unit } \\
\text { Operations }\end{array}$ & 7.16 & 1.37 & 13.25 & 2.26 & 13.60 & 1.61 & 15.63 & .000 & .978 & .332 & 21.36 & .000 \\
\hline $\begin{array}{l}\text { Facilitate } \\
\text { interpersonal \& } \\
\text { organizational } \\
\text { communication }\end{array}$ & 7.38 & 1.69 & 13.36 & 1.92 & 13.58 & 1.65 & 15.11 & .000 & .680 & .499 & 19.04 & .000 \\
\hline Self-Development & 9.67 & 1.32 & 19.38 & 1.86 & 18.69 & 2.74 & 30.18 & .000 & 1.591 & .117 & 23.19 & .000 \\
\hline Total & 73.05 & 6.93 & 132.2 & 7.65 & 131.10 & 8.74 & 39.78 & .000 & 0.756 & .453 & 38.94 & .000 \\
\hline
\end{tabular}

Table (5) Correlation between head nurses managerial innovation knowledge and their professional competency throughout program phases

\begin{tabular}{|c|c|c|c|c|c|c|}
\hline \multirow{4}{*}{$\begin{array}{c}\text { Head nurses' } \\
\text { managerial } \\
\text { innovation } \\
\text { knowledge score }\end{array}$} & \multicolumn{6}{|c|}{ Head nurses' professional competency } \\
\hline & \multicolumn{2}{|c|}{ Pre program } & \multicolumn{2}{|c|}{ Post program } & \multicolumn{2}{|c|}{ Follow up } \\
\hline & $\mathbf{r}$ & P-value & $\mathbf{R}$ & P-value & $\mathbf{r}$ & P-value \\
\hline & 0.08 & 0.41 & 0.65 & $0.002 * *$ & 0.24 & $0.032 *$ \\
\hline
\end{tabular}


Table (6) Correlation between head nurses managerial innovation skills scores and their professional competency throughout program phases

\begin{tabular}{|c|c|c|c|c|c|c|}
\hline \multirow{3}{*}{$\begin{array}{c}\text { Head nurses' } \\
\text { managerial } \\
\text { innovation Skills }\end{array}$} & \multicolumn{6}{|c|}{ Head nurses' professional competency } \\
\cline { 2 - 8 } & \multicolumn{2}{|c|}{ Pre program } & \multicolumn{2}{|c|}{ Post program } & \multicolumn{2}{|c|}{ Follow up } \\
\cline { 2 - 8 } & $\mathbf{r}$ & P-value & $\mathbf{R}$ & P-value & $\mathbf{r}$ & P-value \\
\cline { 2 - 7 } & 0.02 & 0.98 & 0.81 & $0.001 * *$ & 0.33 & $0.029 *$ \\
\hline
\end{tabular}

\section{Discussion}

In the current age of digitalization and globalization, having creativity and innovativeness as a critical attribute in head nurses is becoming a mainstream demand for service and hospitality organizations. Innovation is one of the most important factors for organizational growth. Also human capital plays a significant role in the successful process of innovation. Organizational change occurs when innovative ideas are implemented and translated into the everyday life of healthcare organization, so head nurses must possess innovation skills, which should be considered the "new normal" in order to enhance their professional competencies.

This study aimed to assess the effect of managerial innovation educational program for head nurses on their professional competency through; assessing head nurses knowledge and skills regarding to managerial innovation throughout the program, assessing professional competency of head nurses throughout the program, designing and implementing educational program for managerial innovation for head nurses, investigating effect of managerial innovation educational program for head nurses on their professional competency.

The findings of the present study showed that there was a highly statistical difference improvement of head nurses' knowledge regard managerial innovation between pre and post program phase and between preprogram and follow up phase; that is the educational program has an effect on improving of head nurses knowledge regards to managerial innovation.

From the researcher point of view managerial innovation considered attractive new trend related to head nurse and educational program stimulate curiosity of them to learn more and more about this innovative topic. Also the utilizing of teaching approaches can facilitate the interactions and collaboration in the learning process.

This findings agreed with Rashed,(2017) who studied "Designing and implementing managerial innovation skills training program" he reported that there was a statistical significant improvement of nurse managers knowledge related to managerial innovation immediately after implementation of the program.

Similarly Ebrahim,(2018) who studied "Enhancing creativity and change of 
nursing management staff and its influencing on their performance at Benha University Hospital" who reported that there was highly significant improvement in knowledge level of nurse managers. Also these findings was consistent with Ugwuegbu, (2019) who conducted a study on " knowledge management and organizational innovation" he found that there was a statistical significant improvement in knowledge level of managers.

Concerning head nurses managerial innovation skills thorough program phases, the findings of the present study showed that there was a highly statistical significant difference between preprogram and immediately post program phase and between preprogram and follow up program phase related to cognitive flexibility, problem solving, risk taking, innovation, motivation, emotional intelligence, learning, keeping goal and direction, and communication.

From the researcher point of view head nurses need multiple skills to be innovative; developing and implementing head nurses education program is a necessary strategy to gain innovation skills. Head nurses skills are vital to promote healthy work environments, staff performance, staff retention and positive patient outcomes as patient satisfaction and patient safety.

This result was in agreement with Bockova, (2019) who conducted a study on "Innovation management and its position in company" he emphasized that innovation manager must had human skills as communication and motivation.

In the same line the result was supported by Muzzio,(2018) who conducted a study about " Organizational creativity management: discussion elements" he reported that an innovative manager should has certain skills that enable manager to analyze situation from multiple perspectives and take a critical stance regarding established reality and facilitated the emergence of creativity.

In the same line Mietzner \& Kamprath, (2013) who conducted a study on " A competence portfolio for professionals in the creative industries: creativity and innovation management" they reported that each innovator manager should have set of skills which enable managers to be embedded social context and exhibit traits and skills that facilitate innovation.

The results of the present study revealed that the majority of head nurses had a highly statistical significant difference improvement of professional competency related to promote nursing staff retention, facilitate nursing staff development, perform supervisory responsibilities, ensure patient safety \&quality care, facilitate interpersonal \&organizational communication, selfdevelopment.

From the researcher point of view head nurses' professional competency is vital to promoting healthy work environments, staff performance, staff retention and positive patient outcomes. Head nurses' professional competency can greatly influence the success of health care organizations by enabling them to high performance and collaboration in working toward organizational goals.

This results was similar to Titzer, Shirey, Hauck, (2014) who conducted a study on "A nurse manager succession 
planning model with associated empirical outcomes" they reported that there was a significant increase in professional competencies of nurse managers after the training program. In the same respect Goktepe,(2018) who conducted a study on "Development of managerial competencies for first-level nurse managers in Turkey" and revealed that there was improvement in head nurse professional competencies in post program phase than preprogram.

The result of the present study revealed that there was highly positive statistical significant correlation between head nurses' managerial innovation knowledge and their professional competency. From the researcher point of managerial innovation has a significant importance on head nurses' professional competency as managerial innovation promote head nurse's ability to learn, take risky decisions to solve complex problems in clinical areas; all of this can be helpful for head nurses to achieve high professional competency.

The findings were supported by Rahayu et., al (2018) they studied "The professional competency teachers mediate the influence of teacher innovation and emotional intelligence on school security" they revealed that there was a significant direct relationship exists between teacher innovation and professional competency.

Also this result was similar to Zarefard, (2017) who conducted a study on "Relationship between Entrepreneur's' managerial competencies and innovation start-up intention in university students: an Iranian case" he found positive relationship between managers innovation knowledge and their competency.
The result of the present study revealed that there was highly positive statistical significant correlation between head nurses' managerial innovation skills and their professional competency.

From the researcher point of view maintaining and enhancing managerial innovation skills for head nurses is a critical aspect in promoting their professional competencies as innovative head nurse has good understanding of internal and external work environment, how to deal with complex situations and able to perform tasks effectively and efficiently and that in turn promote and enhance their professional competency. This finding supported the hypotheses of the present study.

This result is supported by Kaya, (2015) who conducted a study on "Linking core competence, innovation and firm performance" and revealed that enhancement of managerial innovation skills will reflect positively on managerial competencies.

The result of the present study demonstrated that there was a highly positive statistically significant correlation between head nurses' managerial innovation knowledge score with their age at post program and there was a positive statistically significant relation between head nurses' managerial innovation knowledge score with their experience at post program.

\section{Conclusion}

Based on the findings of the current study, it can be concluded there was improvement in level of knowledge of the studied head nurses about managerial innovation; minority of head nurses had adequate level of knowledge at preprogram phase while it increased to more than two 
third at post program and follow up study phases respectively compared to preprogram phase. there was improvement in level of skills of the studied head nurses about managerial innovation ; more than two third of head nurses had high level on managerial innovation skills at post program and follow up phases respectively as compared to preprogram phase.

There was improvement in level of the studied head nurses professional competency; more than two third of head nurses had good professional competency at post program and follow up phases respectively as compared to preprogram phase. In addition to there was a highly positive statistical significant correlations between level of knowledge and level of skills, level of knowledge and level of professional, and the level of skills and level of professional competency of the studied head nurses at immediate post and follow up phases of the study.

\section{Recommendations}

1. Hospital management can organize education programs for refreshing and increasing knowledge and skills about nurses' innovation as well as emphasize managerial innovation skills of head nurses.

2- $\quad$ Provide climate and environment to serve inspiration of innovative ideas.

4- Putting an effective system of financial and incorporeal incentives for head nurses focus on reward for the innovative work to encourage them to be innovative and changeable

5- Hospital management should conduct annual assessment of the head nurses level of managerial innovation that considered a key issue to improve their professional competencies and achieve organizational goals.

\section{References}

Abdelazeem, M. (2015). Emotional intelligence and academic achievement among faculty members at faculty of nursing Beni-Suef university, unpublished Master Thesis, Faculty of Nursing , Zagazig University, Pp150155.

Alzoud, M. (2019). The influence of managerial innovation on firm performance in Jordanian commercial banks. Conference on business management research, University of Ultra Malaysia.

Al Zoubi, M. (2016). The impact of administrative decentralization in administrative creativity applied study in Islamic banks Jordan, 64-66, Available

at www.hrmars.comladmin pics $\backslash 1809 . p d f$, Accessed at 12\11\2020.

American Management Association, (2019). Identifying and removing barriers to innovation. Available at https://www.amanet.org/articles.

Bockova, K., (2019). Innovation management and its position in company, Quality innovation prosperity, 16 (2), 14-20.

Chase, L., (2020). Nurse manager competencies, published doctorate dissertation, University of Iowa, 76-77.

Dacko, Z., \&szczepansk,K. (2014). Managerial competencies and innovations in the company. Available at

https://www.researchgate.net/publicatio $\underline{\mathrm{n} / 307680802}$. Accessed at Jaunuary, 2019 . 
Ebrahim,S. (2018). Enhancing creativity and change of nursing management staff and it's influence on their performance at Benha university hospital. unpublished Doctorate Dissertation, faculty of nursing, Benha University, 200-210.

Edwards, M. (2020). The nature and variety of innovation. International Journal of Innovation Studies, 2 (2), 65-79.

Fukada, M. (2018). Nursing Competency: Definition, Structure and Development, Journal of medical science, 6(4), 354362.

Goktepe, N. (2018). Development of managerial competencies for first level nurse managers in turkey. International journal of caring sciences, 11(2), 10971099.

Gunawan,J.,\& Aungsuroch,Y., (2017). Factors contributing to managerial competence of first-line nurse managers: A systematic review. Available at https://doi.org/10.1111/ijn.12611Citati ons.

Kaya, G., (2015). Linking Core Competence, Innovation and Firm Performance, Journal of business research, 73(3) 614-621.

Kelly, C. (2017). Promoting innovation in healthcare, Future health Journal, 4(2), 121-125.

Knoff, C. (2019). A Call for Nurses to Embrace Their Innovative Spirit, The Online Journal of Issues in Nursing, 24 (1), 99-106.

Maia, C. \& Cristina,I. ( 2015). Managerial skills required by innovative and technology based firms. Latin
American Journal of business management 6(3), Pp 45-69.

Markazi,N.,\& Zargar, S.,\& Rafieib,S.,\& Asghari,A.,\& Ghamchilid,A.,\& Shafiie, M. (2019). Managerial competencies of head nurses: A model and assessment tool, British journal of nursing, 28(1), 30-37.

Mietzner, D., \& Kamprath, M. (2013). A competence portfolio for professionals in the creative industries, Creativity and Innovation Management Journal, 22(3), 280-294.

Muzzio, H. (2018). Organizational Creativity Management: Discussion Elements, RAC, Rio de Janeiro, 22 ( 6), 6-11.

Rabea, H. (2017). Nursing Managers' Competency and their Problem Solving Styles at Mansoura University Hospital, Unpublished thesis master. Faculty of nursing, Mansoura university.

Rahayu , S.,\& Ulfatin ,N., \&Wiyono,B., \& Imron, A., Wajdi, M. (2018). The Professional Competency Teachers Mediate the Influence of Teacher Innovation and Emotional Intelligence on School Security, Journal of Social Studies Education Research, 17(4), 6470.

Rashed, M. (2017). Designing and implementing managerial innovation skills training program. Unpublished doctorate dissertation. Faculty of nursing. Assuit university, 13-19.

Saleh,M.,\&Al-Dhaafri, H. (2019). The effect of innovation and strategic planning on enhancing organizational performance of Dubai Police, Available

at 
https://www.emerald.com/insight/2515 -8961.htm. Accessed in 12\12\2019.

Titzer, J.,\& Shirey, M., \& Hauck, S. (2014). A nurse manager succession planning model with associated empirical outcomes, Journal of Nursing Administration, 44(1), 37-46.

Ugwuegbu, C. (2019). Knowledge management and organizational innovation, Strategic, Journal of Business and Social Science (SJBSS), 2(2), 19-26.

Wately, B. (2016). Effects of Innovation Training on Innovative Work Behaviors. Doctor of Philosophy in Organizational Leadership. School of Business \& Leadership. Regent University.

Zarefard, M. (2017). Relationship between Entrepreneur's' managerial competencies and innovation start-up intention in university students: an Iranian case, International Journal of Entrepreneurship , 21 (3), 319-327. 
تأثير برنامج تعليمى عن الابداع الاداري لرؤساء التمريض علي كفائتهم المهنية فاطمة فؤاد السيد- نيرمين محم حسين عيد- فوزية فاروق السيد كامل

تواجه منظمات الرعاية الصحية تحديات غير مسبوقة لتحسين الجودة والحد من الضرر. و يحتاج

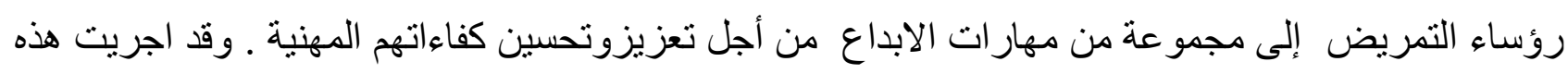

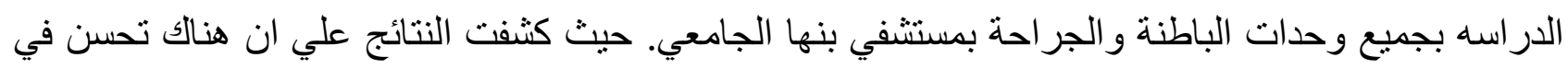

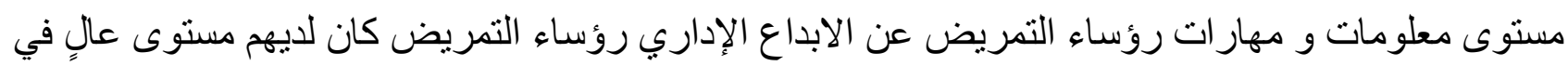

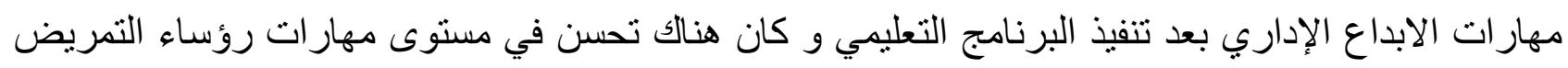

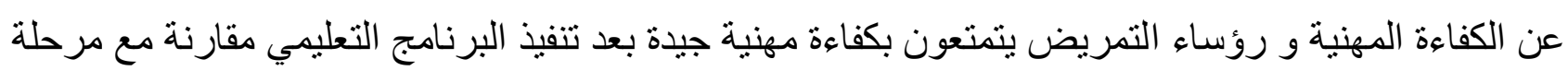

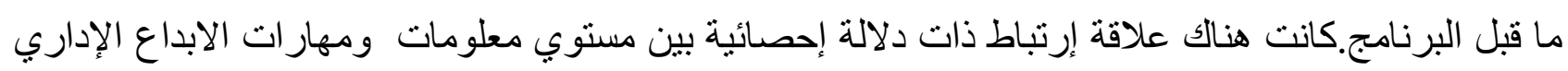

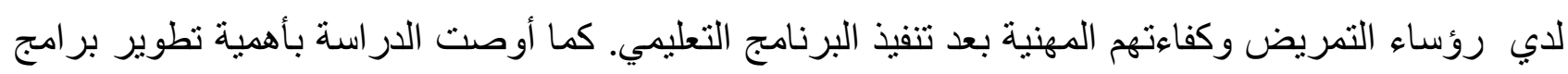
تدريبية لتثقيف رؤساء التمريض عن كيفية تطبيق الإبداع والابتكار في مكان العمل. 